patients with prostate cancer, better tools are required to help choose the appropriate treatment strategy in these individuals.

Original article Fall K et al. (2007) Prostate-specific antigen levels as a predictor of lethal prostate cancer. J Natl Cancer Inst 99: 526-532

\section{Low calcium intake increases the risk of osteoporosis in prostate cancer patients}

Researchers from Spain have revealed a significant relationship between a low daily calcium intake (DCl) and the risk of osteoporosis in men with prostate cancer.

Androgen deprivation therapy (ADT) is the standard treatment for metastatic prostate cancer, and loss of bone mineral density (BMD) leading to osteoporosis is a widely reported side-effect of ADT. To investigate the relationship between $\mathrm{DCl}$ and $\mathrm{BMD}$, Planas et al. analyzed the results of a standard questionnaire filled out by prostate cancer patients who had undergone bone densitometry. Of the 372 participants, 106 had clinically localized prostate cancer treated by radical prostatectomy and were free of biochemical progression, and 266 had clinically advanced disease but no bone metastases, and were treated with ADT.

In total, $93 \%$ of patients were found to have a $\mathrm{DCl}$ below the recommended level ( $\geq 1000 \mathrm{mg} /$ day). The rate of osteoporosis was significantly higher in patients undergoing ADT compared with those treated by radical prostatectomy (54.9\% vs 34.9\%; $P<0.001)$. Mean $\mathrm{DCl}$ did not significantly differ according to ADT status. However, DCl was significantly lower in patients with osteoporosis than in those without (609.7 mg vs $682.8 \mathrm{mg} ; P<0.001$ ), regardless of hormonal status. Multivariate analysis revealed DCI $(P<0.001)$, patient age $(P<0.02)$, and ADT status $(P<0.003)$ and duration $(P<0.001)$ to be independent risk factors for the development of osteoporosis.

These findings support the recommended optimum $\mathrm{DCl}$ of $>1000 \mathrm{mg} /$ day in patients with prostate cancer. The authors recommend that BMD is assessed before initiating ADT and monitored throughout treatment.

Original article Planas J et al. (2007) The relationship between daily calcium intake and bone mineral density in men with prostate cancer. BJU Int 99: 812-816

\section{Dyspareunia can affect patients with interstitial cystitis}

Pelvic pain or pain during sexual intercourse is a clinical feature of interstitial cystitis (IC), but is not characteristic and might be misdiagnosed as endometriosis, vulvodynia, or vulvar vestibulitis. Ottem and colleagues, therefore, used the Female Sexual Function Index (FSFI) to assess whether IC is associated with sexual dysfunction.

The authors enrolled 75 consecutive patients with newly diagnosed IC and 22 asymptomatic controls. Self-reported dyspareunia, mean daily voided volume (not including the first morning volume), and Pelvic Pain and Urgency/Frequency score were compared between these two groups and between women with IC with or without dyspareunia. All participants had been sexually active within the past month.

A significantly greater proportion of patients with IC than controls reported dyspareunia (54 vs 1 ) and had higher Pelvic Pain and Urgency/ Frequency scores (18 vs 3 ) and lower voided volumes $(169 \mathrm{ml}$ vs $294 \mathrm{ml}$; all $P<0.0001)$. Adjusted FSFI scores were lower among patients with IC than among controls $(20.2 \pm 9.6$ vs $29.9 \pm 6.3 ; P<0.0001)$. Overall, 51 patients with IC had abnormally low FSFI scores (<26.55) compared with 3 controls $(P<0.001)$. Patients with IC scored all FSFI parameters (desire, arousal, lubrication, orgasm, satisfaction, pain) lower than controls. All parameters, except pain, were similar in patients with IC who did not report dyspareunia and in those with IC who did.

The authors conclude that all domains of female sexual dysfunction affect patients with IC. They suggest that taking a voiding history might help to differentiate these patients from those with dyspareunia from other causes.

Original article Ottem DP et al. (2007) Interstitial cystitis and female sexual dysfunction. Urology 69: 608-610

\section{Microvascular invasion is an independent predictor of survival in patients with RCC}

Localized renal cell carcinoma (RCC) recurs in a considerable proportion of patients following local treatment. An understanding of prognostic 